\title{
SHORT COMMUNICATION: THE ROLE OF DIFFERENT BODY-RELATED VISUAL CUES IN SYMCHRONIZED TRAMPOLLINING
}

\author{
Thomas Heinen, ${ }^{\mathrm{A}, \mathrm{D}}$ Natalie Czogalla ${ }^{\mathrm{B}, \mathrm{C}}$ \\ University of Hildesheim, Institute of Sport Science, Hildesheim, Germany

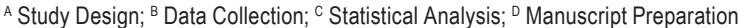 \\ Address for coprespondence: \\ Thomas Heinen \\ University of Hildesheim, Institute of Sport Science \\ Universitaetsplatz 1, 31141 Hildesheim, Germany \\ E-mail: thomas.heinen@uni-hildesheim.de
}

\begin{abstract}
Ahstract. Visual information is thought to be a dominant information source when performing whole-body movements in gymnastics. Visual information can furthermore facilitate performance when being engaged in situations of interpersonal coordination that may occur in sports such as synchronized trampolining. The question arises, which body-related visual cues are most important in the emergence of behavioral synchronization between two gymnasts in synchronized trampolining? To address this question, advanced gymnasts were asked to synchronize their performance to video-sequences of a model gymnast, while body-related visual cues of the model gymnast were systematically manipulated. Results revealed, first that biological motion cues are more important than nonbiological motion cues, and second that the perceived motion of the arms seems to drive synchronicity in synchronized trampolining. It is stated, that biological visual information of the arms is a dominant visual information source when performing leaps in synchronized trampolining.
\end{abstract}

Key Worlls: visual perception, trampoline gymnastics

\section{Introduction}

Perception and action are tightly coupled in the performance of complex skills in sports (Raab, de Oliveira, Heinen, 2009; Warren, 2006). When a gymnast performs leaps on the trampoline, the perception of the trampoline bed in relation to the position of the gymnast when airborne supports the estimation of landing on the trampoline bed, and thus can help to prepare the subsequent leap (Hondzinski, Darling, 2001; Luis, Tremblay, 2008). Perceptual information can furthermore facilitate athlete's performance when being engaged in situations of interpersonal coordination that may occur in sports such as synchronized trampolining (Heinen, Koschnick, Schmidt-Maaß, Vinken, 2014; Schmidt, Fitzpatrick, Caron, Mergeche, 2010). However, the question arises which body-related perceptual cues are most important in the emergence of synchronicity between two gymnasts in synchronized trampolining? 
Behavioral synchronization occurs in rather simple interpersonal coordination tasks (Schmidt, Turvey, 1994), more complex everyday tasks (Richardson, Marsh, Isenhower, Goodman, Schmidt, 2007; Schmidt et al., 2010), as well as in more complex sport-specific tasks (Vesper, van der Wel, Knoblich, Sebanz, 2013). From a theoretical point of view, it is argued that the emergence of interpersonal coordination strongly relies on shared internal representations between action perception, action planning, and action execution (Blake, Shiffrar, 2007; Prinz, 1997; Sebanz, Knoblich, 2009). Thus, in synchronized trampolining, behavioral synchronization may occur when both gymnasts are connected on an informational level. Each gymnast utilizes his/her internal representations of the task at hand to predict the movements of the other actor with the aim of achieving a high degree of behavioral synchronization (Sebanz, Knoblich, 2009).

Visual information is thought to be a dominant source of information when performing skills such as leaps or somersaults (Heinen, 2011; Raab et al., 2009). It is thought that human's visual system is most sensitive to biological motion thereby utilizing different visual cues depending on aspects such as level of expertise as well as on the skill to be performed when performing complex tasks in sport (Blake, Shiffrar, 2007; Mann, Williams, Ward, Janelle, 2007). In synchronized trampolining, two trampolines are placed side by side and two gymnasts perform the same routine at the same time (Kelly, 2014). Both gymnasts could (peripherally) see each other's body movements during performance, and both gymnasts could also hear each other's sounds when being in contact with the trampoline bed during a leap. Given the task constraints in synchronized trampolining, gymnasts are coupled on an informational level and may use this information directly in order to coordinate their own movements with the movements of the gymnast on the other trampoline (Schmidt et al., 2010; Withagen, Michaels, 2005).

In synchronized trampolining, visual information content is first and foremost composed of body-related visual cues of the gymnast on the second trampoline, as well as his/her body movements, and his/her current state of motion. Nevertheless, it is questionable, which body-related visual cues are most important in the emergence behavioral synchronization between two gymnasts in synchronized trampolining? In order to address this question, body-related visual cues of the gymnast on the second trampoline were systematically manipulated by utilizing an occlusion paradigm (Magill, 2007). Gymnasts were asked to synchronize their performance to a model gymnast. It was hypothesized that full visual information should lead to the best degree of behavioral synchronization when performing straight leaps. Systematically occluding body-related visual cues should hamper synchronicity. There was no assumption on which visual cue/s may be most important in the emergence of behavioral synchronization but we sought to explore the role of different body-related visual cues in behavioral synchronization. It was additionally expected that visual information containing biological information should enhance synchronicity as compared to visual information containing no biological information.

\section{Material and Methods}

\section{Participants}

$\mathrm{N}=17$ trampoline gymnasts participated in this study. Gymnasts were active gymnasts with at least seven years of experience in training and competition (age: $17 \pm 2$ years). It was decided to recruit advanced gymnasts to assure that they were already attuned to the relevant visual cues that guide their action (Vickers, 2007). Gymnasts were informed about the general purpose and the procedures of the study. They had normal or corrected-to-normal vision and gave their written consent prior to the study. The study was carried out according to the local universities' ethical guidelines. 


\section{Task and Measures}

Movement Task. The movement task was to perform straight leaps on a trampoline in six experimental conditions. The trampoline was arranged, as it would have been in an international competition in synchronized trampolining, with elevated safety mats in front and behind the trampoline as well as along both sides of the trampoline. The trampoline was placed next to a curtain that was let down from the ceiling of the gymnasium. A data projector was placed behind the trampoline in a way that a full-size video sequence of a gymnast performing straight leaps on a trampoline could be projected on the curtain, thereby reflecting the perspective the gymnast would have had when performing synchronized leaps together with a second gymnast (see Figure 1).

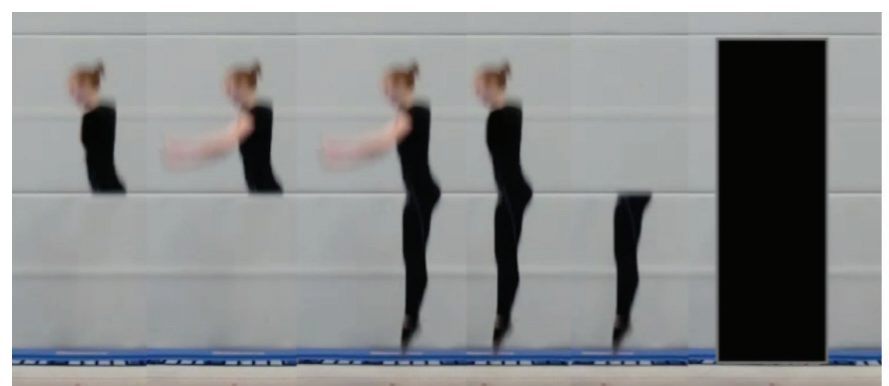

a)

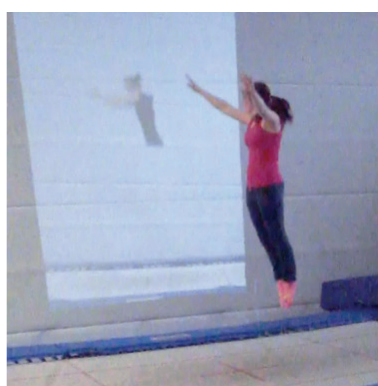

b)

Figure 1. a) Screenshot of the six experimental conditions in this study (from left to right): 1 - occlusion of legs and arms, 2 - occlusion of legs, 3 - full visual information, 4 - occlusion of arms, 5 - occlusion of head, trunk, and arms, 6 - black rectangle. b) Photograph of the procedure of the study: The video sequence of the model was projected to a silver screen on the right side of the gymnast, mimicking the perspective when performing leaps with a partner in synchronized trampolining

A video sequence of a model gymnast was played back on the curtain in each of the six experimental conditions. The task of the gymnast was to adapt his/her leaps to the leaps performed by the model gymnast. He/ she was asked to verbally indicate as soon as he/she subjectively felt to perform synchronously with the video sequence of the model gymnast. After that, he/she should perform another 15 leaps on the trampoline, which were used for later data analysis (see below).

Manipulating Visual Cues. The first experimental condition reflected gymnasts' natural situation in synchronized trampolining. In another four experimental conditions body-related visual cues were systematically occluded. This resulted in the following five conditions: 1 - occlusion of legs and arms, 2 - occlusion of legs, 3 - full visual information, 4 - occlusion of arms, 5 - occlusion of head, trunk and arms. An additional experimental condition comprised a black rectangle. The rectangle contained no body-related visual cues but only reflected the temporal coordination of the leaps.

Temporal Analysis. Gymnasts' temporal coordination was assessed by means of a digital video camera that was placed $15 \mathrm{~m}$ away from the trampoline at an angle of approximately $45^{\circ}$ towards the middle of the trampoline bed (sampling rate: $240 \mathrm{~Hz}$ ). The camera was arranged in a way that it was able to picture the feet of the gymnast when being in contact with trampoline bed together with the model video sequence projected on the curtain. 
From the 15 leaps that were defined by the gymnast as being performed synchronously (see above), another ten leaps were used for further analysis. The absolute values for touchdown times of gymnasts' feet as well as model's feet on the trampoline bed during ten leaps were analyzed. Each touchdown was defined as the first visible contact of gymnasts' and models' feet with the trampoline bed. The mean absolute temporal error between gymnasts' and models' touchdown times was calculated as an indicator of synchronicity (Magill, 2007). An absolute temporal error of 0 would have reflected perfect synchronicity between the gymnast and the model, whereas the larger the temporal error value, the less synchronous the leaps between gymnast and model were.

\section{Procedure}

In the first phase of the study, each individually tested gymnast arrived at the gymnasium, was informed about the general purpose and procedure of the study, and completed the informed consent form. The gymnast was given a 20-minute warm-up phase during which he/she was allowed practice trials of straight leaps on the trampoline (Enoka, 2002). In the second phase the gymnast was asked to adapt his/her leaps to the leaps performed by the model gymnast projected as a video sequence on the silver screen (see Temporal Analysis). The full visual information condition was always presented at first to the gymnast. The other five experimental conditions were presented in a randomized order. Gymnasts were allowed to take breaks as requested. The third phase of the study took place after a gymnast completed all experimental conditions. The gymnast was debriefed and received a small gift for participation.

\section{Data Analysis}

A significance criterion of $a=5 \%$ was defined for all reported results. In order to test the main hypothesis, an analysis of variance with repeated measures was calculated including the mean absolute temporal error between gymnasts' and models' touchdown times as dependent variable. Experimental condition ( 1 - no legs and no arms, 2 - no legs, 3 - full visual information, 4 - no arms, 5 - no head, no trunk and no arms, 6 - black rectangle) was treated as independent variable. Cohen's $f$ was calculated as an effect size (Cohen, 1988).

\section{Results}

It was hypothesized that full visual information should lead to the best synchronicity when performing straight leaps in synchronized trampolining. Systematically occluding visual cues should hamper synchronicity. There was no assumption on which visual cue/s may be most important in the emergence of behavioral synchronization but we sought to explore the role of different body-related visual cues in behavioral synchronization. It was additionally expected that visual information containing biological information should enhance synchronicity.

Results revealed a significant main effect of Experimental Condition on mean absolute temporal error, $F(5,80)=2.36, p<0.05$, Cohen's $f=0.38$ (see Figure 2). 


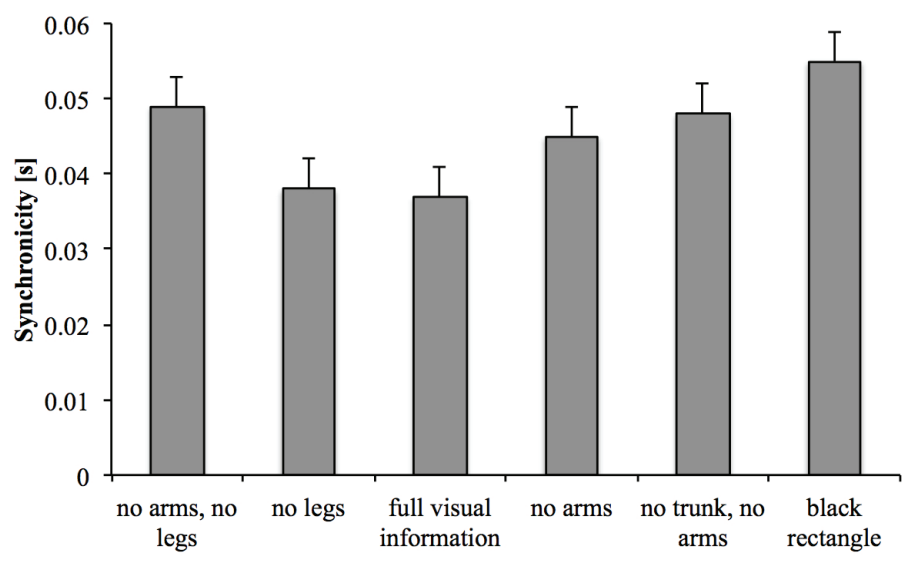

Figulp 2. Mean absolute temporal errors reflecting synchronicity $( \pm S E)$ between gymnast and model in the experimental conditions. The lower the values, the more synchronously the leaps between gymnasts and model were

Gymnasts exhibited best synchronicity in both, the full visual information condition and in the condition where the legs were occluded but the arms were visible. Conditions in which the arms were occluded comprised worse synchronicity, and the black rectangle condition comprised worst synchronicity.

\section{Discussion}

In this study, the role of different body-related visual cues in the emergence of synchronicity in synchronized trampolining was assessed. Gymnasts were asked to synchronize their performance to video-sequences of a model gymnast, while body-related visual cues of the model gymnast were systematically manipulated. Taking the results of the current study together it becomes apparent, first that biological motion cues are more important than non-biological motion cues in synchronized trampolining. Gymnasts exhibited the worst degree of behavioral synchronization when they adapted their leaps to a black rectangle that only reflected the model gymnast's temporal coordination of the leaps but comprised no further body-related visual information. Second, the perceived motion of the arms seems to drive behavioral synchronization in synchronized trampolining. Gymnasts exhibited the best degree of synchronicity in experimental conditions where the arms of the model were visible.

The visual system of humans is highly sensitive to the perception of biological information implying a tight coupling between observation and performance of the same action, which might relate to some common representation of observation (perception) and action, and thus enhance the use of the corresponding information (Blake, Shiffrar, 2007). In synchronized trampolining, behavioral synchronization may benefit from the perceived biological motion of each gymnast thereby utilizing his/her internal representations of the task at hand to predict to movement of the other gymnast (Sebanz, Knoblich, 2009). When referring to the different body-related visual cues of the gymnast on the other trampoline, particularly the perceived motion of the arms seems to drive behavioral synchronization in synchronized trampolining. The arms are thought to be at least partly responsible for a finetuning of vertical impulse when performing leaps (Enoka, 2002). Perceiving the dynamics of the arms in relationship with other visual cues could thus lead to a better estimation of the flight phase, which may help one gymnast to 
adapt his/her leaps to the other gymnast. Additionally, the movement of the arms of the gymnast on the second trampoline may be more salient to the gymnast on the first trampoline due to their greater spatio-temporal dynamics as compared to the movement of the legs or the trunk.

There are several practical implications of this study and one should be highlighted. Achieving a high degree of behavioral synchronization is a key element in synchronized trampolining because it is an important judging criterion, and therefore directly related to successful performance (Freeman, 2007). As a consequence, beginning trampoline gymnast's attention should be directed towards the arms as a relevant informational cue in the emergence of behavioral synchronization in synchronized trampolining. This could be achieved by integrating different forms of perceptual training into skill acquisition processes (Abernethy, Wann, Parks, 1998). Either highlighting (e.g., by using point-lights) or occluding (e.g., by using background-colored clothing) could be fruitful approaches to direct and/or focus learner's attention to different visual cues in synchronized trampolining.

Taken the results of the current study together, it is stated, that biological visual information of the arms is a dominant information cue in the emergence of behavioral synchronization in synchronized trampolining.

\section{References}

Abernethy, B., Wann, J., Parks, S. (1998). Training perceptual-motor skills for sport. In: B. Elliott (ed.), Training in sport. Applying sport science (pp. 1-68). New York, NY: John Wiley \& Sons.

Blake, R., Shiffrar, M. (2007). Perception of human motion. Annual Review of Psychology, 58, 47-73.

Cohen, J. (1988). Statistical power analysis for the behavioral sciences (2nd ed.). New York, NY: Lawrence Erlbaum.

Enoka, R.M. (2002). Neuromechanics of human movement (3rd ed.). Champaign, IL: Human Kinetics.

Freeman, S. (2007). Trampolining. London, UK: A \& C Black Publishers.

Heinen, T. (2011). Evidence for the spotting hypothesis in gymnastics. Motor Control, 15 (2), 267-284.

Heinen, T., Koschnick, J., Schmidt-Maaß, D., Vinken, P.M. (2014). Gymnasts utilize visual and auditory information for behavioral synchronization in trampolining. Biology of Sport, 31 (3), 223-226.

Hondzinski, J.M., Darling, W.G. (2001). Aerial somersault performance under three visual conditions. Motor Control, 5 (3), 281-300.

Kelly, J. (2014). Over \& above: trampoline gymnastics towards world class performance. Oxford, UK: Meyer \& Meyer Sports.

Luis, M., Tremblay, L. (2008). Visual feedback use during a back tuck somersault: evidence for optimal visual feedback utilization. Motor Control, 12 (3), 210-218.

Magill, R.A. (2007). Motor learning and control. Concepts and applications. New York, NY: McGraw-Hill.

Mann, D.T.Y., Williams, A.M., Ward, P., Janelle, C.M. (2007). Perceptual-cognitive expertise in sport: a meta-analysis. Journal of Sport and Exercise Psychology, 29 (4), 457-478.

Prinz, W. (1997). Perception and action planning. European Journal of Cognitive Psychology, 9 (2), 129-154.

Raab, M., de Oliveira, R.F., Heinen, T. (2009). How do people perceive and generate options? In: M. Raab, H. Hekeren, J.G. Johnson (Eds.), Progress in brain research: Vol. 174. mind and motion: The bidirectional link between thought and action (pp. 49-59). Amsterdam, NL: Elsevier.

Richardson, M.J., Marsh, K.L., Isenhower, R.W., Goodman, J.R.L., Schmidt, R.C. (2007). Rocking together: dynamics of intentional and unintentional interpersonal coordination. Human Movement Science, 26 (6), 867-891.

Schmidt, R.C., Fitzpatrick, P., Caron, R., Mergeche, J. (2010). Understanding social motor coordination. Human Movement Science, 30 (5), 834-845.

Schmidt, R.C., Turvey, M.T. (1994). Phase-entrainment dynamics of visually coupled rhythmic movements. Biological Cybernetics, 70 (4), 369-376.

Sebanz, N., Knoblich, G. (2009). Prediction in joint action: what, when, and where. Topics in Cognitive Science, 1, 353-367.

Vesper, C., van der Wel, R.P.R.D., Knoblich, G., Sebanz, N. (2013). Are you ready to jump? Predictive mechanisms in interpersonal coordination. Journal of Experimental Psychology: Human Perception \& Performance, 39 (1), 48-61. 
Vickers, J.N. (2007). Perception, cognition, and decision training. The quiet eye in action. Champaign, IL: Human Kinetics.

Warren, W.H. (2006). The dynamics of perception and action. Psychological Review, 113 (2), 358-389.

Withagen, R., Michaels, C.F. (2005). The role of feedback information for calibration and attunement in perceiving length by dynamic touch. Journal of Experimental Psychology: Human Perception \& Performance, 31 (6), 1379-1390.

Cite this article aS: Heinen, T., Czogalla, N. (2016). Short Communication: The Role of Different Body-Related Visual Cues in Synchronized Trampolining. Central European Journal of Sport Sciences and Medicine, 13 (1), 15-21. DOI: 10.18276/cej.2016.1-02. 
